\title{
Differences in Pathogenicity, Genetic Variability and Cultivar Responses among Isolates of Fusarium oxysporum from Strawberry in Australia
}

\author{
Michelle L. Paynter ${ }^{1}$ \\ Department of Agriculture and Fisheries, Maroochy Research Facility, 47 Mayers Road Nambour, \\ Queensland, Australia 4560; and School of Agriculture and Food Sciences, The University of \\ Queensland, Saint Lucia, Queensland, Australia 4072 \\ Elizabeth Czislowski \\ School of Agriculture and Food Sciences, The University of Queensland, Saint Lucia, Queensland, \\ Australia 4072
}

\author{
Mark E. Herrington \\ Department of Agriculture and Fisheries, Maroochy Research Facility, 47 Mayers Road Nambour, \\ Queensland, Australia 4560 \\ Elizabeth A.B. Aitken \\ School of Agriculture and Food Sciences, The University of Queensland, St. Lucia, Queensland, \\ Australia 4072
}

\begin{abstract}
Additional INDEX words. elongation factor 1 alpha, Fragaria $\times$ ananassa, F. oxysporum f. sp. fragariae, fusarium wilt, vegetative compatibility groups

Abstract. Variation in the virulence of Fusarium oxysporum $\mathrm{f}$. sp. fragariae (Fof $)$ strains is important when evaluating the resistance of plants to this fungus. Twenty-five isolates of $F$. oxysporum harvested from strawberry (Fragaria $\times$ ananassa) plants growing in Australia were characterized using pathogenicity tests, vegetative compatibility groups (VCGs), and genetic analysis of translation elongation factor 1 alpha (EF-1 $\alpha)$. The level of disease varied depending on isolate used, indicating heterogeneous populations of $F o f$. Two distinct VCGs were identified and corresponded to two of the 10 lineages identified by partial EF-1 $\alpha$. Using a subset of $F$ of isolates, resistance in eight cultivars ranged from highly resistant to highly susceptible, with some cultivar $\times$ isolate interaction. 'Strawberry Festival', 'QHI Sugarbaby', and 'DPI Rubygem' had high levels of resistance across all isolates. Isolates from Western Australia (WA) were genetically distinct from those from Queensland (QLD) and were more virulent to 'Camarosa', a major cultivar grown in WA.
\end{abstract}

Fusarium wilt of strawberry is caused by Fof and is a major soil-borne disease of strawberry worldwide. This disease quickly kills infected plants by impeding water and nutrient movement in the vascular tissues of the plant. Since the introduction of the fumigant methyl bromide in the 1970s, and the use of disease-free transplants, losses due to fusarium wilt and other soil-borne disease have been minimized. However, with the phase out of methyl bromide and no commercially viable equivalent on the market soil-borne diseases have recently caused major losses in the Australian strawberry industry.

Fusarium oxysporum is associated with a group of pathogens causing crown and root diseases of strawberry. These include: Colletotrichum species, Macrophomina phaseolina, Phytophthora species, Pythium species, Rhizoctonia fragariae, and Verticillium species. Crown and root diseases of strawberry are considered a serious problem to strawberry production with $10 \%$ of plant death in QLD and up to $50 \%$ in the Perth district of

Received for publication 22 July 2016. Accepted for publication 3 Oct. 2016. This project has been funded by Horticulture Innovation Australia Limited using the strawberry industry levy and funds from the Australian Government. The Queensland Government has also cofunded the project through the Department of Agriculture and Fisheries.

This paper is a portion of a Master's degree submitted by Michelle Paynter. ${ }^{1}$ Corresponding author. E-mail: michelle.paynter@daf.qld.gov.au.
WA (Fang et al., 2012; Golzar et al., 2007; Phillips and Golzar, 2008) being attributed to Fof. In WA, high incidences of plant decline and death were reported in strawberry crops in 2005 in both fumigated and nonfumigated soils. In some fields 'Camarosa' and 'Gaviota' suffered up to 60\% mortality (Golzar et al., 2007). As a result, surveys were implemented to identify the causes and severity of the problem. Fof was identified as a predominant pathogen responsible for much of the plant deaths. In 2005, Fof was isolated from $80 \%$ to $90 \%$ of the samples tested (Phillips and Golzar, 2008). In 2008, a survey undertaken to determine the severity of crown and root diseases in WA showed that plant decline and death occurred in both nonfumigated and fumigated fields. Fusarium oxysporum was the dominant pathogen isolated from crowns. Up to $41 \%$ of the isolates tested were $F$. oxysporum, isolated either as the sole species or one of several species isolated from infected crowns and roots (Fang et al., 2011).

Queensland is the biggest producer of strawberries in Australia, growing mainly for the domestic market. In 2014-15, up to 30\% of isolations undertaken from plants showing root and crown disease at the Department of Agriculture and Fisheries, QLD, have shown $F$. oxysporum (A. Gomez, unpublished data).

An increase in disease outbreaks of fusarium wilt in the past few years, plus the search for alternative disease management has placed emphasis on the development of resistant cultivars. 
Knowledge of the diversity of the pathogen is critical to ensure that potential new cultivars are tested against a wide a range of pathotypes. However, information on the genetic variation of Fof across Australia is unknown. An assessment of a broad range of Australian isolates and their pathogenicity would allow the identification of highly virulent or diverse strains and give some indication of the diversity of Australian isolates. In addition, virulent isolates can be used to challenge potential breeding lines.

Molecular techniques and VCG testing have been used to resolve genetic variation amongst Fof isolates. Vegetative compatibility tests are used for characterizing variation in genetically isolated asexual populations. Isolates belonging to the same VCG, confirmed by the ability to form a prototrophic heterokaryon, can exchange genetic information and are often clonally derived from populations of a forma specialis (Correll et al., 1987; Kistler et al., 1991). In Korea, Hyun and Park (1996) used random amplified polymorphic DNA (RAPD) polymerase chain reactions (PCRs) to separate 24 isolates into two distinct clades and assigned 32 isolates into four major VCGs. Nagarajan et al. (2004) also used RAPD and restriction fragment length polymorphism of the intergenic spacer region of ribosomal DNA to study variation amongst isolates in Korea. They identified eight distinct clusters, and noted dissimilarities between isolates from different sites. Nagarajan et al. (2006) identified three major VCGs and one incompatible group among 22 isolates, and noted a relatively high correlation existed among VCG and RAPD, and virulence.

A number of studies have shown the nuclear translation EF$1 \alpha$ gene region can reveal genetic relationships amongst formae speciales of $F$. oxysporum. O'Donnell et al. (1998) demonstrated that EF-1 $\alpha$ could resolve relationships within $F$. oxysporum. Fourie et al. (2009) determined the relatedness amongst VCGs of $F$. oxysporum f. sp. cubense and other formae speciales and nonpathogens using EF-1 $\alpha$ data. These studies reveal that the EF-1 $\alpha$ gene regions can separate isolates of $F$. oxysporum.

The first objective of this study was to evaluate pathogenicity and variation amongst $F$. oxysporum isolates from strawberry in Australia by means of pathogenicity testing, VCGs, and partial sequencing of the EF- $1 \alpha$ gene region. The second objective was to evaluate cultivar responses to Fof. This information will help us understand the diversity of isolates and their potential use in screening cultivars and breeding lines.

\section{Materials and Methods}

Fungal isolates and plant material. Twenty-eight $F$. oxysporum isolates from Australia were used in this study (Table 1). Twenty-seven of the isolates were obtained from the crowns and roots of strawberry plants showing symptoms of crown and root rot disease. The isolates were identified as $F$. oxysporum based on spore and colony morphology. The majority of isolates came from southeast QLD and from WA where heavy infestations have been reported (Golzar et al., 2007). Singlespore isolates were stored on filter paper at Maroochy Research Facility, QLD, Australia. One isolate of F. oxysporum f. sp. zingiberi, Foz_N39298, was obtained from The Department of Primary Industries and Fisheries Plant Pathology Herbarium, Brisbane, Australia, and included in molecular studies only as a comparator.

Strawberry transplants were obtained from a certified nursery. These plants were placed in pots containing steamsterilized river sand and coir (1:1) fertilized at 2-week intervals
Table 1. Australian isolates of Fusarium oxysporum used in comparing pathogenicity in strawberry, vegetative compatibility groups, and elongation factor $1 \alpha$ sequences. Foz_N39298 was included in molecular studies only as a comparator.

\begin{tabular}{|c|c|c|c|c|}
\hline Accession no. $^{\mathrm{z}}$ & BRIP no. ${ }^{y}$ & Yr collected & Geographic origin $^{\mathrm{x}}$ & $\mathrm{VCG}^{\mathrm{n}}$ \\
\hline N9054 & $62023 a$ & 1962 & Victoria Point, QLD & HSI \\
\hline N9055 & $62107 \mathrm{a}$ & 1962 & Victoria Point, QLD & HSI \\
\hline SA126 & $62105 \mathrm{a}$ & 1981 & Adelaide Hills, SA & SM \\
\hline N9103 & $62025 \mathrm{a}$ & 1989 & Nambour, QLD & SM \\
\hline N9551 & $62267 \mathrm{a}$ & 1990 & Palmwoods, QLD & HSI \\
\hline N10010 & $62049 a$ & 1991 & Caboolture, QLD & HSI \\
\hline N10226 & $62028 \mathrm{a}$ & 1992 & Hastey Park, NSW & HSI \\
\hline $\mathrm{N} 13581^{v}$ & $62122 a$ & 2002 & Palmview, QLD & A \\
\hline N15309" & $62106 a$ & 2005 & Wamuran, QLD & A \\
\hline $\mathrm{N} 15457^{\mathrm{v}}$ & $62124 a$ & 2005 & Nambour, QLD & A \\
\hline N15915 & $62042 a$ & 2006 & Wamuran, QLD & A \\
\hline $\mathrm{N} 16004^{\mathrm{v}}$ & $62076 \mathrm{a}$ & 2006 & Wamuran, QLD & SM \\
\hline N16239 & $62109 a$ & 2006 & Wanneroo, WA & B \\
\hline N16240 & $62108 \mathrm{a}$ & 2006 & Wanneroo, WA & B \\
\hline N16818 & $62077 \mathrm{a}$ & 2007 & Nambour, QLD & SM \\
\hline N16893 & $62061 \mathrm{a}$ & 2007 & Beenleigh, QLD & SM \\
\hline N16999 & $62029 a$ & 2007 & Redlands, QLD & SM \\
\hline N17203 & $62097 \mathrm{a}$ & 2008 & Stanthorpe, QLD & SM \\
\hline $\mathrm{N} 17337^{\vee}$ & $62051 \mathrm{a}$ & 2008 & South Perth, WA ${ }^{\mathrm{u}}$ & B \\
\hline N17350 & $52120 \mathrm{~b}$ & 2008 & Chevallum, QLD & SM \\
\hline N18419 & & 2009 & Stanthorpe, QLD & $\mathrm{n} / \mathrm{t}$ \\
\hline $\mathrm{N} 18421^{\mathrm{v}}$ & & 2009 & Stanthorpe, QLD & $\mathrm{n} / \mathrm{t}$ \\
\hline N18437 & $62125 \mathrm{a}$ & 2009 & Stanthorpe, QLD & SM \\
\hline $\mathrm{N} 18462^{\mathrm{v}}$ & $53862 a$ & 2009 & Wanneroo, WA & B \\
\hline N18440" & $62027 \mathrm{a}$ & 2009 & Nambour, QLD & HSI \\
\hline N18842 & $62053 a$ & 2009 & Stockleigh, QLD & SM \\
\hline N18936 & $62094 a$ & 2009 & Atherton, QLD & SM \\
\hline Foz_N392988 & $39298 \mathrm{a}$ & 1998 & Beerwah, QLD & $\mathrm{n} / \mathrm{t}$ \\
\hline
\end{tabular}

${ }^{\mathrm{z}}$ All isolates were obtained from the Department of Agriculture and Fisheries, Queensland government, Brisbane, Australia.

${ }^{y}$ Herbarium code for the Queensland Department of Primary Industries Plant Pathology Herbarium, Brisbane, Australia.

${ }^{\mathrm{x}} \mathrm{QLD}=$ Queensland, SA = South Australia, NSW $=$ New South Wales, $\mathrm{WA}=$ Western Australia.

${ }^{\mathrm{w}} \mathrm{VCG}=$ vegetative compatibility group, HSI = heterokaryon selfincompatible, $\mathrm{SM}=$ single-member, $\mathrm{n} / \mathrm{t}=$ not tested.

'Isolates used in virulence testing.

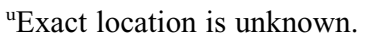

with 23N-4P-14K soluble fertilizer (Aquasol; Yates, Padstow, Australia), at the recommended rates.

All statistical analyses for pathogenicity tests were performed using Genstat (version 11.1; VSN International, Hemel Hempstead, UK). Analyses of pathogenicity of isolates, and cultivar responses, were performed using severity rating means and compared by analysis of variance on the estimate of Fisher's protected least significant difference test $(P=0.05)$. Cultivar by isolate disease response over time was investigated using a mixed model longitudinal analysis incorporating cubic smoothing splines in ASReml-R (Butler et al., 2009), following the approach of Verbyla et al. (1999).

Pathogenicity tests. A sample of nine isolates (N13581, N15309, N15457, N16004, N17337, N18419, N18421, $\mathrm{N} 18462$, and N18440) were used for root dip inoculations with the susceptible cultivar Kabarla (D. Hutton, unpublished data) in March. To validate the procedure, N18462, from WA was used as a positive control; it had been isolated from affected 
plants in an episode associated with a high incidence of crown rot in the 2005 and 2006 seasons in WA.

Isolates were plated onto 1/4-strength potato dextrose agar (PDA), and incubated in the dark at $27^{\circ} \mathrm{C}$ for 3 weeks. The spores were collected from the plates following addition of sterile deionized water by rubbing the agar surface with a glass spreader. The spore suspension was then filtered through four layers of cheesecloth. The conidial concentration was determined using a haemocytometer and adjusted to $1 \times 10^{6}$ conidia $/ \mathrm{mL}$.

Six-month-old plants were removed from their pots, the crown and roots washed, drained, and then immersed in the inoculum for $10 \mathrm{~min}$ in random order, with five plants per isolate treatment. Five untreated control plants were immersed in sterile water. The plants were potted into pots containing steam-sterilized river sand and coir (1:1). About $10 \mathrm{~mm}$ of sterile gravel was added to the top of the soil to prevent splash. Each pot was then randomly allocated, onto a heated bench at $28^{\circ} \mathrm{C}$ in a glasshouse with natural light.

Disease assessment. Disease development was monitored weekly and visual ratings taken after 8 weeks. The severity of foliar symptoms was assessed on a 0 to 10 visual disease index, where $0=$ plant healthy, with erect growth and full vigor; $1=$ plant healthy, with a smaller canopy and moderate vigor; $3=$ plant with a slight wilt, with the lower leaves affected; $5=$ plant with a moderate wilt, with the mature leaves collapsing but young leaves healthy; $7=$ plant with a severe wilt, with most of the plant collapsed and mature leaves desiccated; $9=$ plant with a very severe wilt, with the entire plant collapsed and most of plant desiccated; and $10=$ plant dead. A mean disease severity score for each isolate treatment was calculated across replicates. The degree of virulence $(x)$ of $F$ of was determined from the mean disease severity rating by the following scale: $x \leq 2=$ nonvirulent; $2<x \leq 4=$ slightly virulent; $4<x \leq 7=$ moderately virulent; and $x>7=$ highly virulent.

Crowns were sampled for the recovery of Fof on two to five plants of each isolate treatment after 8 weeks to confirm successful Fof infection. The crowns were washed and surface sterilized in $0.5 \%$ sodium hypochlorite for $5 \mathrm{~min}$, and rinsed three times in sterile water. The crowns were cut in cross sections and discolored pieces (or if healthy, section of vascular tissue) plated onto 1/4-strength PDA and incubated at $27^{\circ} \mathrm{C}$. After 1 week, the plates were inspected for the presence of Fof.

To confirm that the isolations obtained from the plants were responsible for Fof infection and subsequent plant symptoms, the re-isolates were each tested on six plants of 'Kabarla' in June. Six noninoculated plants were treated as controls. In addition, six plants were treated with the original N17337 isolate as a comparison.

VEGETATIVE COMPATIBILITY GROUPING OF ISOLATES. Twentyfive isolates were used in vegetative compatibility tests as described by Puhalla (1985) and Correll et al. (1987). The isolates were plated onto $1 / 4$-strength PDA, and incubated at $27^{\circ} \mathrm{C}$ for 1 week. Two to three biochemically different nitrate nonutilizing mutants (nit mutants) were obtained from each isolate using minimal medium (MM) amended with potassium chlorate (Puhalla, 1985). Sectors growing from the restricted colonies were classified by aerial mycelium, identified by phenotype produced when a small mycelial disk taken from the edge of the sector was transferred onto $\mathrm{MM}$ with one of three forms of a nitrogen source, $\mathrm{NaNO}_{2}, \mathrm{NaNO}_{3}$, and hypoxanthine in the medium and termed nit 1 , nit 3 , and Nit $\mathrm{M}$, respectively. All combinations of mutants were paired on MM in petri dishes. Two isolates were paired by placing a mycelial disk of one nit mutant of an isolate in the center with four nit mutants of another isolate arranged around the edges. Where possible, a Nit $\mathrm{M}$ was paired with up to four nit 1 mutants from each isolate pair. Where a Nit $\mathrm{M}$ had not been generated, a nit 3 was substituted. If able to form a prototrophic heterokaryon with the same isolate but different phenotypic class (nit mutant), isolates were considered heterokaryon self-compatible, if unable to form a heterokaryon they were considered heterokaryon self-incompatible (HSI). Combinations of nit mutants from all isolates were paired to verify VCGs, determined by the isolate's ability to form a heterokaryon. If an isolate formed no heterokaryon with any other isolate it was termed a "single member."

PCR AND PARTIAL SEQUenCING OF EF-1 $\alpha$. For DNA extraction, isolates were plated onto 1/4-strength PDA and incubated at $27^{\circ} \mathrm{C}$ for 1 week. About $50 \mathrm{mg}$ of mycelia from each isolate was extricated and collected using a sterile scalpel blade, cooled in liquid nitrogen and ground into a powder. DNA extraction was performed immediately using a DNeasy Plant Mini Kit (Qiagen, Victoria, Australia) according to the manufacturer's instructions.

The EF- $1 \alpha$ region was amplified using forward and reverse primers EF-1 and EF-2 (O'Donnell et al., 1998) (Table 2). PCR amplification was performed using a thermal cycler (Mastercycler ep; Eppendorf, Hamburg, Germany), in $25.0 \mu \mathrm{L}$ reaction volumes containing 12.5 $\mu \mathrm{L}$ MyTaq Red Mix (Bioline, Eveleigh, Australia), $0.25 \mu \mathrm{L}(50 \mu \mathrm{M})$ of each primer (Sigma-Aldrich, Castle Hill, Australia), and 1.0 $\mu \mathrm{L}$ DNA template. The thermocycling conditions were $94{ }^{\circ} \mathrm{C}$ for $3 \mathrm{~min}$, followed by 35 cycles of $94{ }^{\circ} \mathrm{C}$ for $45 \mathrm{~s}, 55^{\circ} \mathrm{C}$ for $15 \mathrm{~s}$, and $72{ }^{\circ} \mathrm{C} 15 \mathrm{~s}$, and a final extension at $72{ }^{\circ} \mathrm{C}$ for $5 \mathrm{~min}$. A negative control (no DNA) was included. PCR products were visualized on $1.5 \%$ agarose gel stained with ethidium bromide to confirm expected product size (600-700 bp).

The EF-1 $\alpha$ PCR product was sequenced in both directions using primers EF-3 (internal forward) and EF-22T (internal reverse) (Table 2). PCR product purification and sequencing were performed by Macrogen Inc. (Seoul, Korea). Sequence quality was confirmed by using both EF-1 and EF-2, and by visual inspection of the chromatogram using Finch TV (Geospiza, Seattle, WA).

Phylogenetic analysis. Following alignment of sequences, consensus sequences for each isolate were produced at $678 \mathrm{bp}$. To understand the associations and similarity of the isolates, a phylogenetic tree was 
constructed using $F$. oxysporum EF-1 $\alpha$ nucleotide sequences. Consensus sequences for EF-1 $\alpha$ of $F$. oxysporum isolates were produced using Geneious (version 7.1; Biomatters, Auckland, New Zealand) and aligned and edited using ClustalW in Geneious. For the EF-1 $\alpha$ alignment, 26 isolates were used. Two sequences of EF-1 $\alpha$ from Fof isolates downloaded from GenBank were also included as comparisons, Maff744009 from Japan and KJ776745.1 from Turkey, and an EF-1 $\alpha$ sequence of $F$. verticillioides $(F v)$ (KF466424.1) (GenBank) was used to root the data set.

The tree was inferred using the maximum likelihood method based on the general time reversible model (Nei and Kumar, 2000). The initial trees for the heuristic search were obtained by applying the neighbor-joining method to a matrix of pairwise distances estimated using the maximum composite likelihood approach. Bootstrap analyses were made with 1000 replications. Evolutionary analyses were conducted in MEGA6 (Tamura et al., 2013).

Cultivar Responses to FoF. Isolates were plated onto 1/4strength PDA and incubated in the dark at $27{ }^{\circ} \mathrm{C}$ for $\approx 3$ weeks for inoculum preparation (as described previously). 'Camarosa', 'Earliblush', 'Festival', 'Fortuna', 'Kabarla', 'Redlands Joy', 'Rubygem', and 'Sugarbaby' were inoculated with four virulent isolates N15309, N13581, and N18440 from QLD and N18462 from WA at a concentration of $1 \times 10^{6}$ conidia $/ \mathrm{mL}$. 'Kabarla' (susceptible) and 'Festival' (resistant) were used as standards and 'Camarosa' was included due to recent and severe losses to this cultivar in WA. A completely randomized design was used with nine plant replicates of each cultivar and six non-inoculated 'Kabarla' plants. In addition, six plants of 'Kabarla' (susceptible control) and six 'Festival' plants (resistant) were used as untreated controls and similarly immersed in sterile water only. Ratings for disease severity were taken at 10 time points (weeks 3 to 12 postinoculation). The plants were inoculated in October and crowns sampled for recovery of Fof as described previously.

\section{Results}

Pathogenicity testing. Disease development in the susceptible 'Kabarla' varied amongst isolates. Symptoms included chlorosis and necrosis of the leaves, lesions on the petioles, wilting and collapse of petioles and leaves, and plant death. The earliest symptoms occurred after 3 weeks. The isolates were classified as pathogenic based on the disease severity index, from $0=$ healthy plant to $10=$ plant dead (Table 3 ). Isolates with ratings $>2$ were designated as Fof or of the forma specialis fragariae, and included N13581, N15309, N17337, and N18462, which were highly virulent, and N15457 and N18440, which were less virulent (Table 3). N16004, N18419, and N18421 typically resulted in healthy plants with clean crowns, and were comparable to the controls. As there was no significant difference between the controls, these isolates were regarded as nonpathogenic.

The level of discoloration in the vascular tissues was consistent with the severity of disease in the leaves. Plants showing foliar symptoms had vascular discoloration and crown rots, whereas those showing no symptoms had clean crowns and nondiscolored tissues. Crown isolations carried out on symptomatic plants produced colonies of $F$. oxysporum. Fusarium oxysporum was re-isolated from all crown samples with the exception of plants inoculated with N16004, N18419, and N18421, and one plant inoculated with N18440.

To confirm that these isolations were pathogenic the reisolates underwent further tests. The isolates included N13581, N15309, N15457, N17337, and N18440, and were given accession numbers N13581a, N15309a, N15457a, N17337a, and N18440a. At 6 weeks after inoculation all the plants showed symptoms. The disease was worse on plants inoculated with N15309a, N13581a, and N17337a. There was a significant difference between these isolates and N15457a and N18440a (Table 3). Fusarium oxysporum was isolated from the crowns of symptomatic plants. All the controls were symptom free and no Fof was recovered.

VEGETATIVE COMPATIBILITY GROUPING OF THE ISOLATES. Three to 25 nitrate utilization mutants were generated from each of 25 isolates when cultured on MM containing potassium chlorate. Typically nit 1 mutants were produced, with fewer nit 3 and Nit M. All isolate sectors produced at least one nit 3 or Nit $\mathrm{M}$ to be used as testers.

Table 3. Means of visual rating for disease severity (where $0=$ healthy plant and $10=$ plant dead), on cultivar Kabarla inoculated with Fusarium oxysporum isolates harvested from diseased strawberry in Australia. Ratings taken at 8 weeks post-inoculation from pathogenicity experiment.

\begin{tabular}{|c|c|c|c|c|}
\hline Isolate & Mean primary disease rating $(0-10 \text { scale })^{\mathrm{z}}$ & Re-isolate code & Mean disease rating $(0-10 \text { scale })^{y}$ & $\begin{array}{c}\text { Pathogenicity } \\
\text { rating }^{\mathrm{x}}\end{array}$ \\
\hline- & - & N17337 (comparison) & $8.17 \mathrm{~d}$ & Pathogenic \\
\hline N18421 & $0.6 \mathrm{a}^{\mathrm{w}}$ & $\mathrm{n} / \mathrm{t}^{\mathrm{v}}$ & $\mathrm{n} / \mathrm{t}$ & Nonpathogenic \\
\hline N18419 & $1.4 \mathrm{a}$ & $\mathrm{n} / \mathrm{t}$ & $\mathrm{n} / \mathrm{t}$ & Nonpathogenic \\
\hline N18440 & $2.0 \mathrm{ab}$ & N18440 a & $4.0 \mathrm{~b}$ & Pathogenic \\
\hline N15457 & $3.4 \mathrm{~b}$ & N15457 a & $5.7 \mathrm{c}$ & Pathogenic \\
\hline N18462 & $7.8 \mathrm{~cd}$ & $\mathrm{n} / \mathrm{t}$ & $\mathrm{n} / \mathrm{t}$ & Pathogenic \\
\hline N17337 & $9.2 \mathrm{~d}$ & N17337 a & $9.3 \mathrm{~d}$ & Pathogenic \\
\hline N13581 & $9.6 \mathrm{~d}$ & N13581 a & $9.0 \mathrm{~d}$ & Pathogenic \\
\hline $\mathrm{SED}=0.934^{v}$ & & $\mathrm{SED}=0.807$ & & \\
\hline
\end{tabular}

${ }^{\mathrm{z}} 0=$ healthy plant and $10=$ plant dead $(\mathrm{n}=5)$.

${ }^{\mathrm{y}} \mathrm{n}=6$.

${ }^{\mathrm{x}}$ Isolates considered pathogenic if a mean disease rating $\geq 2$.

${ }^{\text {w}}$ Means with same letter are not significantly different at $P=0.05$; least significant difference $=1.8$ and 1.6.

${ }^{\mathrm{v}} \mathrm{n} / \mathrm{t}=$ not tested, SED $=$ standard error of difference. 


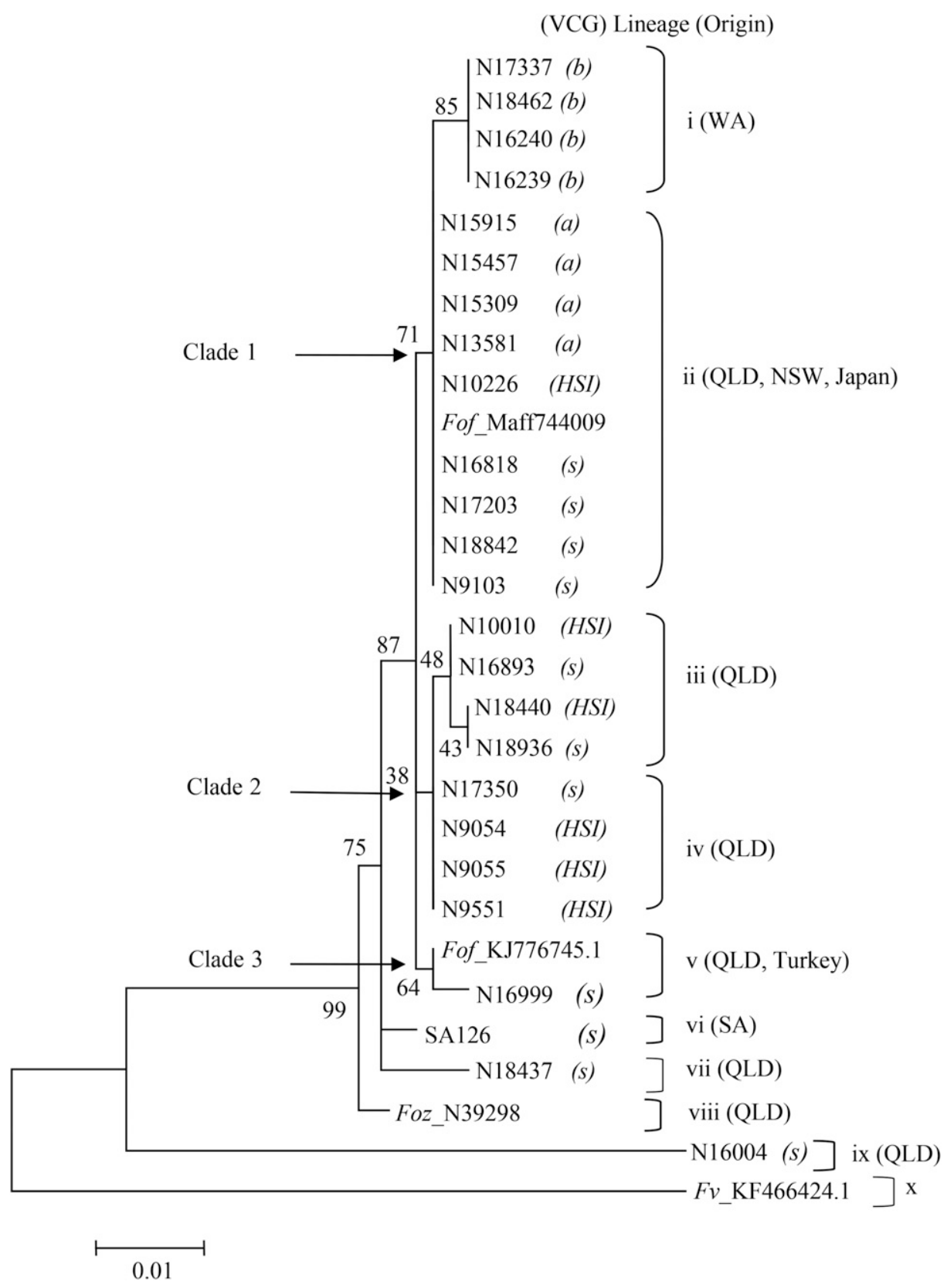

Fig. 1. The phylogenetic tree of 26 elongation factor $1 \alpha(\mathrm{EF}-1 \alpha)$ sequences (Table 1) rooted with Fusarium verticillioides ( $F$ v_KF466424.1) inferred by the maximum likelihood method based on the general time reversible model (Nei and Kumar, 2000). Foz_N39298 and N16004 were included as outgroups, and the publically available EF-1 $\alpha$ sequences Fof_Maff744009 and Fof_KJ776745.1 were included as comparisons. Numbers at the nodes represent bootstrap values from 1000 replications. The tree is drawn to scale with branch lengths measured in the number of substitutions per site. Evolutionary analyses were conducted in MEGA6 (Tamura et al., 2013). Ten clonal lineages are identified by (i to $\mathrm{x}$ ), and the three clades are indicated by arrows next to the corresponding interior branches. Corresponding vegetative compatibility groups (VCGs a, b), heterokaryon self-incompatible (HIS) and single-member (SM) isolates are shown next to isolate number in parenthesis. Origins of isolates are shown next to Lineage in parenthesis; NSW $=$ New South Wales, WA $=$ Western Australia, QLD = Queensland.

All mutants of an individual isolate were compatible with each other with the exception of N9054, N9055, N9551, N10010, N10226, and N18440, which were therefore designated as HSI. Four isolates from the Sunshine Coast, QLD (N13581, N15309, N15457, and N15915), formed prototrophic heterokaryons with each other and were designated as VCGa. Similarly, the four isolates from WA (N16239, N16240, N17337, and N18462) produced well-formed heterokaryons with each other and were designated as VCGb. The remaining 11 isolates were considered singlemember VCGs, as they were unable to form a heterokaryon with another isolate (Table 1).

Sequence And Phylogenetic ANALYSIS. Phylogenic analysis of EF-1 $\alpha$ sequences of the isolates showed that the 29 sequences grouped into 10 lineages. Twenty-four isolates grouped into three clades (Fig. 1). Clades 1 to 3 comprised five lineages (i to $\mathrm{v}$ ), representing isolates from QLD, New South Wales (NSW) and WA, and also included the publically available Japanese sequence (Fof_Maff744009). There was no variation detected among the four EF-1 $\alpha$ sequences in lineage (i), all of which were from WA and comprised VCGb. Isolates from lineage (ii) were all closely associated and occurred with highest frequency, representing $34 \%$ of all isolates. Lineage (ii) included isolates from QLD, NSW, and Japan, grouped into VCGa, as well as four single isolates and one HSI. The lineages (iii) to (v) comprised isolates of HSI and singlemember VCGs, and originated from QLD, SA, and the publically available Fof_KJ776745.1 sequence from Turkey. The outgroup Foz_N39298 (Fusarium zingiberi), was separated from the Fof isolates, the only exception being N16004 which was even more distantly related with the other Fof isolates than Foz_N39298.

Cultivar Responses to FoF. Twelve weeks after inoculation all plants of 'Camarosa' $\times$ N18462, the majority of plants of 'Fortuna', 'Earliblush', and 'Kabarla' $x$ N18462, and plants of 'Kabarla' $\times$ N13581, showed strong disease symptoms. Plants of 'Fortuna' were moderately susceptible overall isolates, while 'Festival' and 'Sugarbaby' were resistant (Table 4). Isolate N18462 (from WA) resulted in more severe disease expression on most cultivars except Festival and Rubygem with zero ratings for this isolate. There were significant cultivar effects after 3 weeks, and then significant cultivar $\times$ isolate interaction after 7 weeks. N18440 and N15309 only produced significant disease levels on 'Fortuna', whereas N13581 only produced significant disease levels on 'Kabarla'. Fof was re-isolated from the crowns of symptomatic plants. Significant colonies of $F$. oxysporum confirmed effective inoculation. All the controls were healthy and free of symptoms, with no internal discoloration. 
Table 4. Means of visual rating for disease severity (where $0=$ healthy plant and $10=$ plant dead), from eight cultivars of strawberry inoculated with four Fusarium oxysporum f. sp. fragariae isolates harvested from diseased strawberry in Australia. Ratings taken at 12 weeks postinoculation.

\begin{tabular}{llccc}
\hline Cultivar $\times$ isolate & N13581 & N15309 & N18440 & N18462 \\
\hline Camarosa & $1.0 \mathrm{a}^{2}$ & $0.1 \mathrm{a}$ & $1.1 \mathrm{a}$ & $10.0 \mathrm{~d}$ \\
Earliblush & $0.1 \mathrm{a}$ & $0.4 \mathrm{a}$ & $0.0 \mathrm{a}$ & $8.2 \mathrm{~d}$ \\
Festival & $0.0 \mathrm{a}$ & $0.0 \mathrm{a}$ & $1.1 \mathrm{a}$ & $0.0 \mathrm{a}$ \\
Fortuna & $2.4 \mathrm{abc}$ & $3.9 \mathrm{bc}$ & $4.3 \mathrm{c}$ & $4.3 \mathrm{c}$ \\
Kabarla & $4.6 \mathrm{c}$ & $1.1 \mathrm{a}$ & $0.0 \mathrm{a}$ & $7.8 \mathrm{~d}$ \\
Redlands Joy & $0.0 \mathrm{a}$ & $0.0 \mathrm{a}$ & $0.0 \mathrm{a}$ & $1.7 \mathrm{ab}$ \\
Rubygem & $1.6 \mathrm{ab}$ & $1.1 \mathrm{a}$ & $0.0 \mathrm{a}$ & $0.0 \mathrm{a}$ \\
Sugarbaby & $0.0 \mathrm{a}$ & $1.1 \mathrm{a}$ & $0.0 \mathrm{a}$ & $1.1 \mathrm{a}$
\end{tabular}

${ }^{\mathrm{z}}$ Means with same letter are not significantly different at the $P=0.05$ level. Standard error of differences: cultivar $=0.6$, isolate $=0.4$, cultivar $\times$ isolate $=1.3$. time there is a need to account for the temporal correlation between repeated measurements. In this analysis, we have modeled the temporal correlation using the approach of Diggle (1988) and De Faveri et al. (2015), implementing a heterogeneous autoregressive covariance structure together with a subject or Plant effect. The analysis showed a significant cultivar $\times$ isolate interaction over time as can be seen in Fig. 2, where predictions at each time have been plotted for each cultivar $\times$ isolate. Predictions for each cultivar $\times$ isolate are also given for week 7 and week 12 in Table 5 together with their respective average standard error of difference.

\section{Discussion}

In this study, the pathogenicity of different isolates of Fof varied significantly. The mean disease rating ranged from nil (no symptoms), to moderate (wilting and some plant death), to severe (the death of most plants), indicating heterogeneity among populations of Fof. Pathogenicity tests undertaken in this study identified six virulent isolates: N13581, N15309, N15457, N17337, N18440, and N18462, of which four were subsequently used to test the susceptibility of different cultivars. Three of the isolates tested were nonpathogenic to strawberry. These isolates were harvested from strawberry plants showing symptoms of crown and root rot, but failed to produce symptoms in our study. This may be due to the several reasons. The isolates may have been harvested along with other pathogens from diseased plants. Many $F$. oxysporum strains are nonpathogenic, limited to the root cortex, and cause no visible symptoms to their host. Alternatively, the isolates may have lost their virulence due to cultural generation caused by frequent sub-culturing on carbohydrate-rich media which is common in $F$. oxysporum species (Leslie et al., 2006).

Isolates were also categorized using complementation analyses (complementation testing of mutants). Assignment of isolates into VCGs was difficult, with 11 isolates belonging to single-member VCGs and six isolates were identified as HSI. This may have been due to problems generating appropriate mutants. However, two distinct VCGs were identified amongst the 25 isolates. The number of single isolate groups (11) is high. Hyun and Park (1996) from Korea identified four major VCGs, with only one of these being a single isolate group.

Fig. 2. Predicted disease development of eight strawberry cultivars after-inoculation with Fusarium oxysporum $\mathrm{f}$ sp. fragariae isolates. The data are plotted over time using a mixed model longitudinal analysis incorporating cubic smoothing splines. Predicted rating for disease development ranged from 0 to 10 , where $0=$ healthy and $10=$ dead

The data were investigated using a mixed model longitudinal analysis incorporating cubic smoothing spines. As the data were obtained by repeatedly measuring the same plants over
Phylogenetic analysis based on EF-1 $\alpha$ sequences showed variability among isolates associated with different regions. For the majority of isolates, obtained from within southeast QLD, 
Table 5. Predicted disease ratings for each of the eight strawberry cultivars inoculated by four Fusarium oxysporum fragariae isolates (cultivar $\times$ isolate) after week 7 and week 12. The predictions are made from the repeated measures analysis across time using cubic smoothing splines (Fig. 2).

\begin{tabular}{|c|c|c|}
\hline \multirow[b]{2}{*}{ Cultivar $\times$ isolate } & \multicolumn{2}{|c|}{ Prediction of disease rating } \\
\hline & Week 7 & Week 12 \\
\hline Camarosa.N13581 & 0.2 & 1.1 \\
\hline Earliblush.N13581 & 0 & 0.1 \\
\hline Festival.N13581 & 0 & 0 \\
\hline Fortuna.N13581 & 1.6 & 2.1 \\
\hline Kabarla.N13581 & 2.4 & 4.9 \\
\hline Redlands Joy.N13581 & 0 & 0 \\
\hline Rubygem.N13581 & 1.2 & 1.5 \\
\hline Sugarbaby.N13581 & 0 & 0 \\
\hline Camarosa.N15309 & 0 & 0.1 \\
\hline Earliblush.N15309 & 0 & 0.4 \\
\hline Festival.N15309 & 0 & 0 \\
\hline Fortuna.N15309 & 2.7 & 3.6 \\
\hline Kabarla.N15309 & 0.8 & 1 \\
\hline Redlands Joy.N15309 & 0 & 0 \\
\hline Rubygem.N15309 & 0.4 & 1.2 \\
\hline Sugarbaby.N15309 & 0.3 & 1.1 \\
\hline Camarosa.N18440 & 0.1 & 1 \\
\hline Earliblush.N18440 & 0 & 0 \\
\hline Festival.N18440 & 0.9 & 1.1 \\
\hline Fortuna.N18440 & 3.3 & 4.1 \\
\hline Kabarla.N18440 & 0 & 0 \\
\hline Redlands Joy.N18440 & 0 & 0 \\
\hline Rubygem.N18440 & 0.2 & 0.1 \\
\hline Sugarbaby.N18440 & 0 & 0 \\
\hline Camarosa.N18462 & 3.2 & 10 \\
\hline Earliblush.N18462 & 2.7 & 8.8 \\
\hline Festival.N18462 & 0 & 0 \\
\hline Fortuna.N18462 & 3.2 & 4.2 \\
\hline Kabarla.N18462 & 5.2 & 7.3 \\
\hline Redlands Joy.N18462 & 0.3 & 1.7 \\
\hline Rubygem.N18462 & 0 & 0 \\
\hline Sugarbaby.N18462 & 0.1 & 1 \\
\hline $\operatorname{avsed}^{z}$ & 1.13 & 1.07 \\
\hline
\end{tabular}

${ }^{\mathrm{z}}$ Average standard error of difference.

there was moderate diversity. In contrast, all of the isolates from WA were of the same genotype and VCG. This limited diversity could be due to sampling only a small area in the Perth district and a wider range of isolates may need to be tested to obtain a better representation of genetic diversity in WA.

The EF- $1 \alpha$ sequences of Fof isolates within clade 1 (Fig. 1) were determined to be closely related and represented individuals not only from QLD, NSW, and WA, but also from Japan (Maff744009). Sequences within clade 2 were also closely related. Fof sequences within clade 3 were genetically dissimilar, but shared a recent common ancestor with clades 1 and 2 . Results of the EF-1 $\alpha$ analysis and pathogenicity testing of isolates indicate that isolates belonging to clades 1 to 3 (lineages $\mathrm{i}$ to $\mathrm{v}$ ) belong in the forma specialis fragariae. The remaining divergent lineages SA126 and N18437 (lineages vi and vii), were concluded to be genetically dissimilar. The isolate N16004 was determined to not be of the forma specialis, nonpathogenic to strawberry fragariae, and belongs to a separate lineage.
The evaluation of strawberry cultivars showed varying degrees of susceptibility to Fof. It is apparent that there are significant differences in isolate virulence to strawberry and also significant differences in cultivar responses to Fof isolates. Fitting cubic smoothing splines (in ASReml) to model the cultivar $\times$ isolate combination disease response provides an idea of disease development over time. Looking at the different disease profiles over time (week 7 and week 12) for each cultivar $\times$ isolate (Table 5), not all cultivars for the four different isolates behave the same and clearly some isolates affect some cultivars differently than others.

The isolate 'N18462' from WA was the most aggressive isolate. In addition, it seems particularly well adapted to attack 'Camarosa', which is very susceptible and which accounts for a high percentage of strawberry planted in WA. However, this isolate caused no disease symptoms on 'Festival' and 'Rubygem'. Field management practices should ensure effective control across the whole production field and additionally avoid the spread of aggressive isolates such as N18462 to new locations.

We are not certain why for some isolates (e.g., N15309, N13581) ratings on cv. Kabarla varied among experiments: for example, N15309 in Table 3 had a rating of 7.2, but in Table 4 had a rating of 1.1. These may be a seasonal effect as the experiments were conducted at different times of the year (March and October). However, the cultivar $\times$ isolate experiment (Table 4) was conducted 3 years after the pathogenicity tests (Table 3) and the fact that the two isolates showing most change (i.e., N15309, N13581) had been sub-cultured more frequently than the others, because these former had been used in additional Fof screening trials, may imply loss of virulence has occurred over time (Leslie et al., 2006). This aspect requires further investigation.

Collectively, the results from pathogenicity testing, VCG and EF- $1 \alpha$ analyses indicate a diverse range of pathogenicity and genotypes occurring in F. oxysporum isolates obtained from the major strawberry production regions of Australia, and these include both pathogenic and nonpathogenic strains. Many of the cultivars tested were found to be very resistant or tolerant to fusarium wilt, indicating that there is a good source of resistance available for use in resistance breeding. This research shows some variation in cultivar susceptibility to different strains of Fof. Some cultivars were resistant to one or more isolates, whereas other cultivars were relatively susceptible. The existence of a potential race structure in $F$. oxysporum is not uncommon; e.g., F. oxysporum cubense (Ploetz and Correll, 1988) and is highly relevant for the development of durable resistant cultivars. As such, isolate choices need to be considered in resistance screenings. This may include screening with local isolates but also regularly benchmarking against more virulent isolates. Additionally in commercial fields changes in the frequency of occurrence of virulent isolates needs to be monitored regularly over time.

\section{Literature cited}

Butler, D., B. Cullis, A. Gilmour, and B. Gogel. 2009. ASReml-R, reference manual. Release 3. Queensland Dept. Primary Ind. Fisheries, Toowoomba, Australia.

Correll, J.C., C.J.R. Klittich, and J.F. Leslie. 1987. Nitrate nonutilizing mutants of Fusarium oxysporum and their use in vegetative compatibility tests. Phytopathology 77:1640-1646. 
Diggle, P. 1988. An approach to the analysis of repeated measurements. Biometrics 44:959-971.

De Faveri, J., A.P. Verbyla, W.S. Pitchford, S. Venkatanagappa, and B.R. Cullis. 2015. Statistical methods for analysis of multi-harvest data from perennial pasture variety selection trials. Crop Pasture Sci. 66:947-962.

Fang, X.L., D. Phillips, H. Li, K. Sivasithamparam, and M.J. Barbetti. 2011. Severity of crown and root diseases of strawberry and associated fungal and oomycete pathogens in Western Australia. Australas. Plant Pathol. 40:109-119.

Fang, X., J. Kuo, M. You, P. Finnegan, and M. Barbetti. 2012. Comparative root colonisation of strawberry cultivars Camarosa and Festival by Fusarium oxysporum f. sp. fragariae. Plant Soil 358:75-89.

Fourie, G., E.T. Steenkamp, R.C. Ploetz, T.R. Gordon, and A. Viljoen. 2009. Evolutionary relationships amongst the Fusarium oxysporum f. sp. cubense vegetative compatibility groups. Appl. Environ. Microbiol. 75:4770-4780.

Golzar, H., D. Phillips, and S. Mack. 2007. Occurrence of strawberry root and crown rot in Western Australia. Australas. Plant Dis. Notes $2: 145-147$.

Hyun, J.W. and W.M. Park. 1996. Differentiation of Fusarium oxysporum $\mathrm{f}$. sp. fragariae isolates by random amplified polymorphic DNA (RAPD) analysis. Korean J. Plant Pathol. 12:41-46.

Kistler, H.C., E.A. Momol, and U. Benny. 1991. Repetitive genomic sequences for determining relatedness among strains of Fusarium oxysporum. Phytopathological 81:331-336.

Leslie, J.F., B.A. Summerell, and S. Bullock. 2006. The Fusarium laboratory manual. Vol. 2, No. 10. Blackwell Publ., Ames, IA.

Nagarajan, G., M.H. Nam, J.Y. Song, S.J. Yoo, and H.G. Kim. 2004. Genetic variation in Fusarium oxysporum f. sp. fragariae populations based on RAPD and rDNA RFLP analyses. Plant Pathol. 20:264-270.

Nagarajan, G., S.W. Kang, M.H. Nam, J.Y. Song, S.J. Yoo, and H.G. Kim. 2006. Characterization of Fusarium oxysporum f. sp. fragariae based on vegetative compatibility group, random amplified polymorphic DNA and pathogenicity. Plant Pathol. 22:222-229.

Nei, M. and S. Kumar. 2000. Molecular evolution and phylogenetics. Oxford Univ. Press, New York, NY.

O’Donnell, K., E. Cigelnik, and H.I. Nirenberg. 1998. Molecular systematics and phylogeography of the Gibberella fujikuroi species complex. Mycologia 90:465-493.

O’Donnell, K., D.A. Sutton, A. Fothergill, D. McCarthy, M.G. Rinaldi, M.E. Brandt, and D.M. Geiser. 2008. Molecular phylogenetic diversity, Multilocus haplotype nomenclature, and In vitro antifungal resistance within the Fusarium solani species complex. J. Clin. Microbiol. 46:2477-2490.

Phillips, D. and H. Golzar. 2008. Strawberry root and crown rot disease survey, 2005 and 2006 seasons. Dept. Agr. Food, Govt. Western Austral., Bul. No. 4747.

Ploetz, R.C. and J.C. Correll. 1988. Vegetative compatibility among races of Fusarium oxysporum f. sp. cubense. Plant Dis. 72:325-328.

Puhalla, J.E. 1985. Classification of strains of Fusarium oxysporum on the basis of vegetative compatibility. Can. J. Bot. 63:179-183.

Tamura, K., G. Stecher, D. Peterson, A. Filipski, and S. Kumar. 2013. MEGA6: Molecular evolutionary genetics analysis version 6.0. Mol. Biol. Evol. 30:2725-2729.

Verbyla, A.P., B.R. Cullis, M.G. Kenward, and S.J. Welham. 1999. The analysis of designed experiments and longitudinal data by using smoothing splines. Appl. Stat. 48:269-312. 